\title{
EDITORIAL \\ Drinking at the end of pregnancy: why don't we see it?
}

\author{
Pediatric Research (2020) 88:142; https://doi.org/10.1038/s41390- \\ 020-0846-1
}

Prenatal alcohol exposure in late pregnancy is rarely studied and this makes the contribution by Umer et al. very useful. ${ }^{1}$ Their study adds to the accumulating data demonstrating that screening dried blood spots can identify biomarkers of prenatal alcohol exposure at the end of pregnancy, which may be more common than previously thought. This study suggests that about 1 in 12 pregnant women in West Virginia are drinking at the end of pregnancy. This is very similar to a study of prenatal alcohol exposure in Texas where 8.4\% of women, also 1 in every 12, were drinking late in pregnancy. ${ }^{2}$

Our interest in drinking during late pregnancy came about after reports from five women who delivered a child were later diagnosed with fetal alcohol spectrum disorder (FASD). These women had completed substance abuse treatment and were in an FASD support group when this topic came up. One woman reported that while in labor and accompanied by her sister they stopped in the hospital parking lot where she drank a standard container of vodka ( $375 \mathrm{ml}$ ) with an alcohol content of $40 \%$ a few minutes before entering the hospital. She felt that this would increase the speed of delivery and decrease the pain. The other four women reported drinking at the onset of labor or while on the way to the hospital. We then had the women sign a record release and give us a copy of their admission, labor, and delivery and discharge notes. We found no mention of alcohol in either the nurse's notes or the physician's notes for these five women or their infants.

We then decided to do a literature review on this topic to see how often women are identified as using alcohol while in labor and delivery. We searched all languages and all years. We identified 12 studies reporting on 16 women who had a positive blood alcohol concentration at delivery and where it was also measured in their infant. ${ }^{3}$ We felt that a positive blood alcohol concentration in the infant eliminated the possibility that they had consumed alcohol in the hospital after delivery. We found that women who are drinking at labor and delivery are rarely reported. ${ }^{3}$ We then estimated that in the United States every year 34,285 women (93 per day) are drinking on the day of delivery. ${ }^{3}$ Thus drinking at the end of pregnancy or during labor is not rare.

However, prenatal alcohol exposure in late pregnancy appears to be rarely reported. We have been asking our obstetric colleagues, neonatologists, and labor and delivery nurses if they can recall cases of alcohol use identified during labor and delivery. They do not. We feel that increased attention to the recognition of alcohol use in pregnancy including late pregnancy is warranted since it provides an opportunity to identify a woman who has a substance use disorder and who needs treatment. Successful treatment has the potential to prevent exposure in subsequent pregnancies and could also reduce the risk of mortality and morbidity associated with prenatal alcohol exposure among these women and their infants. $^{3-5}$

Larry Burd ${ }^{1}$

${ }^{1}$ Department of Pediatrics, University of North Dakota School of Medicine and Health Sciences, 1301 N Columbia Road Stop 9037, Grand Forks, ND 58202-9037, USA Correspondence: Larry Burd (larry.burd@und.edu)

Publisher's note Springer Nature remains neutral with regard to jurisdictional claims in published maps and institutional affiliations.

\section{REFERENCES}

1. Umer, A. et al. Prevalence of alcohol use in late pregnancy. Pediatr. Res. https://doi.org/10.1038/s41390-019-0731-y (2020).

2. Bakhireva, L. N. et al. Prevalence of prenatal alcohol exposure in the state of Texas as assessed by phosphatidylethanol in newborn dried blood spot specimens. Alcohol Clin. Exp. Res. 41, 1004-1011 (2017).

3. Schaff, E., Moreno, M., Foster, K., Klug, M. G. \& Burd, L. What do we know about prevalence and management of intoxicated women during labor and delivery? Glob. Pediatr. Health https://doi.org/10.1177/2333794X19894799 (2019).

4. Burd, L. et al. Mortality rates in subjects with fetal alcohol spectrum disorders and their siblings. Birth Defects Res. A Clin. Mol. Teratol. 82, 217-223 (2008).

5. Schwartz, M. et al. The hidden face of fetal alcohol spectrum disorder. Curr. Womens Health Rev. 13, 96-102 (2017). 